\title{
Intermodulation Distortion in Switched Multibeam Antennas for Cellular Radio Systems
}

\author{
Mattias Wennström, Anders Rydberg and Tommy Öberg \\ Signals and Systems Group, Uppsala University, \\ Box 528, SE-751 20 Uppsala, Sweden, Email:mw@signal.uu.se
}

\begin{abstract}
The performance of switched multibeam antennas for cellular radio systems is investigated when the transmit amplifier is of a multicarrier (MCPA) type. The MCPA nonlinearity generates intermodulation distortion in the transmitted signal that is spatially filtered by the array radiation pattern. Hence, the intermodulation distortion becomes direction-dependent, and the direction of the generated intermodulation products of any order is derived. It is shown by using Monte Carlo simulations how the downlink carrier to interference ratio depends on the frequency reuse distance and on the MCPA linearity measured as its noise power ratio (NPR). For reuse factor one systems, which is made possible by use of multibeam antennas, the linear co-channel interference dominates the interference. On the contrary, for a reuse factor seven system it is shown that a weak nonlinearity directly gives a degradation in carrier to interference ratio (CIR) due to intermodulation distortion. However the reuse factor seven system has a large CIR overhead due to the increase in CIR using a multibeam antenna, and the outage probability is thereby only slightly affected.
\end{abstract}

\section{INTRODUCTION}

The deployment of switched multibeam antennas at base stations (BS) in cellular TDMA/FDMA systems has either shown to improve the capacity or to extend the radio coverage by increasing the carrier to interference ratio (CIR). The reduced interference levels allows for a reduction in frequency reuse distance, thereby increasing the spectrum efficiency of the cellular system [1].

One drawback with the use of array antennas at the BS sites is the increased amount of hardware compared to a conventional BS. For each antenna and for each frequency channel a single carrier power amplifier is required for the downlink transmission. Thus, to reduce size, cost and power consumption of the BS, multicarrier power amplifiers (MCPA) have been suggested for use in multiantenna BS [2]. The co-amplification of several carriers on different frequencies in an MCPA generates intermodulation products (IMP) due to the nonlinearity and due to the non-constant envelope of the combined signal. Often the third order IMP are considered (of type $2 \omega_{j}-\omega_{k}$ and $\left.\omega_{j}+\omega_{k}-\omega_{l}\right)$, as they fall onto other frequency channels that are used in the system and thereby, cannot be removed by filtering.
To meet the requirements in the mobile system specification of allowed spurious emission, linearisation of MCPA:s is required. Although linearisation techniques significantly reduces the intermodulation distortion in the output signal, some residual intermodulation distortion remains, and it is useful to know to what level the intermodulation has to be suppressed by the lineariser, as a lower level implies a more expensive linearisation method. The problem addressed in this paper is how the linearity requirements on the transmit MCPA depends on the frequency reuse distance for a system using switched multibeam antennas at the BS. The linearity of the MCPA is defined by using the noise power ratio (NPR) measured from a standardized NPR test [3].

A cellular system with a total of $C$ frequency channels equally distributed over $K$ cells is assumed in this paper. The $C / K$ frequency channels in each cell are further divided in a sector-trunkpool scheme where each $120^{\circ} \mathrm{sec}-$ tor has a unique set of $C / 3 K$ frequency channels assigned to it. The mobile stations (MS) in the sector will receive interference of two types. First, linear interference comes from BS in the (primarily) first tier of co-channel BS. Secondly, intermodulation interference is generated in the MCPA in the same BS as the MS is connected to, and if the set of frequency channels used in the sector are equally spaced, it can be shown that the generated intermodulation products falls onto the same set of frequencies.

Note that the intermodulation interference is emitted with low power, but the MS is usually close to the BS, hence the signal has a low path loss. Compare with the linear co-channel interference from first tier BS, which is transmitted at high power, but the larger BS to MS distance gives a high path loss. Hence, there is a point in the degree of MCPA linearity, where the major interference at the MS changes from being linear interference, from first tier BS, to intermodulation interference, from "own" BS.

One property of MCPA:s in conjunction with multibeam antennas is that the radiated IMP will be spatially filtered by the beam-pattern of the antenna array [5],[6]. This implies that in some directions the generated IMP will be suppressed by the side lobe level of the multibeam radiation pattern and in other directions, the IMP power will be amplified by the antenna array gain by coherent addition of the IMP from all antennas. Hence, the carrier to interference ratio at the MS in the system depends on the position and beam allocation of the other users in the cell and in neighboring cells. A Monte Carlo simulation is used to estimate the outage probability, which is the probabil- 
ity that the carrier to interference ratio (CIR) for a MS fall below some threshold. The outage probability is evaluated for different MCPA linearities and at different frequency reuse distances.

\section{CO-CHANNEL INTERFERENCE ON THE DOWNLINK}

The frequencies in a cluster are assumed to be planned according to a fixed channel allocation scheme as seen in Fig. 1. Furthermore, each sector is equipped with an $N$ el-

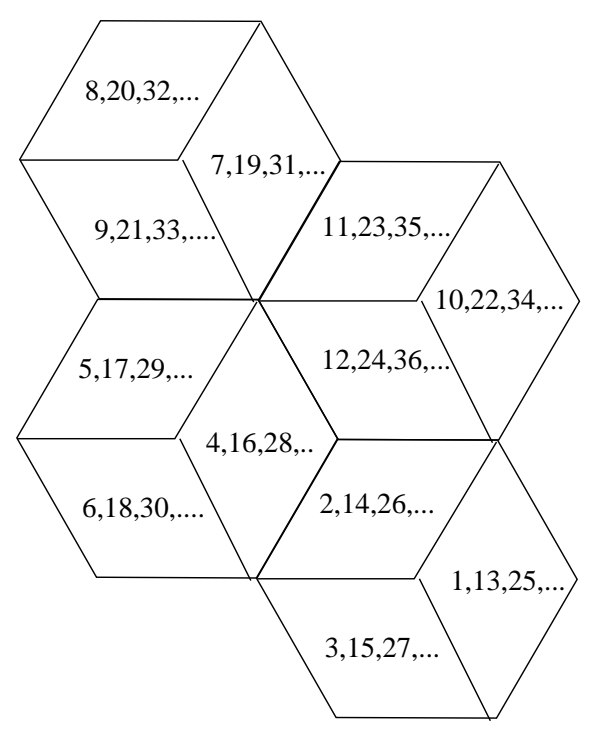

Figure 1: Assignment of frequency channels to sectors in a fixed channel allocation scheme with cluster size $K=4$ cells.

ement antenna array, hence $N$ fixed beams can be formed in each sector and thus $3 N$ beams in each cell. In the BS transmit path, each antenna is preceded by an MCPA with maximum and minimum output power per carrier of 43 and $13 \mathrm{dBm}$ respectively. The power regulation per transmitted carrier is performed in $2 \mathrm{~dB}$ steps and is based on the received signal strength of the uplink signal averaged over the fast multipath fading. Path loss compensation is done for half of the loss, following the ideas in [7]:

$$
P_{E I R P}=B-0.5 \cdot G_{l i n k}
$$

Here, $G_{\text {link }}$ is the MS-BS link gain, that is, the quotient between the received power at the BS and the transmitted power from the MS, which is assumed known and $B$ is constant that has to be adjusted for optimal performance of the system, see [7]. After the power regulation (1), the power is adjusted to be in the interval between minimum and maximum output power.

\section{A. Intermodulation Interference}

Consider a BS with transmit MCPA's operating in it's nonlinear region, close to saturation to maximize the power efficiency of the amplifier. The power of the received IMP at the MS is a function of the array pattern, the nonlinear characteristics of the amplifiers and the spatial distribution of the MS [8]. In the remaining part of the paper, the study is restricted to the third order intermodulation products that falls "in band", i.e. at the frequencies $2 \omega_{i}-\omega_{j}$ and $\omega_{i}+\omega_{j}-\omega_{k}$. This is justified by the fact that these IMP terms contains most of the intermodulation energy.

Furthermore, assume that the frequency channels in Fig. 1 are equally spaced, that is, the center frequencies are related as

$$
\omega_{k}=\omega_{0}+k \Delta \omega
$$

where $\omega_{k}$ is the center frequency of frequency channel $k$, $\Delta \omega$ is the frequency channel separation and $\omega_{0}$ is a reference frequency.

In each sector, the center frequencies of the used frequency channels are related as $\omega_{k+1}=\omega_{k}+3 K \Delta \omega$ where $K$ is the cluster size. It is easy to verify that with this frequency planning, the generated third order IMP falls onto other frequencies used in the downlink in the same sector. For example frequency channel 13 and 25 generates IMP on channel $1\left(2 \omega_{13}-\omega_{25}=\omega_{1}\right)$ which is also used in that sector, see Fig. 1.

\section{B. Calculating the MCPA Output Signal}

If the complex gain of an MCPA as a function of the input power of a single carrier is measured, the AM/AM and $\mathrm{AM} / \mathrm{PM}$ conversion characteristics of the amplifier is obtained. These are commonly used to describe the nonlinearity. When simulating MCPA:s in conjunction with array antennas, each of the IMP in each of the MCPA:s output signal must be separately calculated, because their relative phases and amplitudes will affect the radiation pattern of each IMP. A commonly used method uses the fast Fourier transform (FFT) to yield the output power at arbitrarily frequencies from a nonlinear device with a multi-tone input. With more than one IMP on the same frequency in the output signal, the FFT method fail to distinguish between them, as it gives only the sum of the total IMP on that frequency. Instead, Shimbo [9] proposed a method by fitting the AM/AM and AM/PM characteristics to a Bessel series expansion. With this method it is possible to find an analytical expression for the amplitude and phase of the desired signals and each of the IMP:s in the output of a nonlinear amplifier with a multi-tone input. Hence, this method suits our needs. Shimbo's method is described below.

If $g(A)$ and $f(A)$ are the AM/AM and AM/PM characteristics of the amplifier, find the coefficients $\alpha, b_{s}$ in a Bessel series expansion by solving

$$
\begin{gathered}
\left\{\alpha, b_{1}, \ldots, b_{S}\right\}= \\
\arg \min _{\alpha, b_{1}, \ldots, b_{S}}\left\{\left|g(A) e^{j f(A)}-\sum_{s=1}^{S} b_{p} J_{1}(\alpha s A)\right|^{2}\right\}
\end{gathered}
$$

for all amplitudes $A$ in the input amplitude interval. Here $J_{1}$ is the 1st order Bessel function and note that the coef- 
ficients $b_{s}$ are complex. Assume that the input consists of $M$ phase modulated $\left(\phi_{m}(t)\right)$ carriers with amplitude $A_{m}$ and write the input as

$$
e_{i n}(t)=\sum_{m=1}^{M} A_{m} e^{j\left(\omega_{m} t+\phi_{m}(t)\right)}
$$

where $\omega_{m}$ is the center frequency of frequency channel $m$. The output signal of the MCPA can be written as

$$
e_{o u t}(t)=\sum_{L}\left(M(L)\left\{e^{j \sum_{k=1}^{M} l_{k}\left(\omega_{k} t+\phi_{k}(t)\right)}\right\}\right)
$$

where $L$ is the set

$$
L=\left\{\left\{l_{1}, l_{2}, \ldots, l_{M}\right\}: \sum_{k=1}^{M} l_{k}=1, \sum_{k=1}^{M}\left|l_{k}\right|=1,3\right\}
$$

to restrict the output to the "in band" first and third IMP. Now, define the set $L_{p}$ as

$$
L_{p}=L \cap\left\{\left\{l_{1}, l_{2}, \ldots, l_{M}\right\}: \sum_{k=1}^{M} l_{k} \omega_{k}=\omega_{p}\right\}
$$

to consider the output in the frequency channel with center frequency $\omega_{p}$ only. Using the coefficients from the least square fit in equation (3), the complex voltage gain for the $p$ :th component in the output at frequency $\omega_{p}$ and can be written as [9]

$$
M\left(L_{p}\right)=\sum_{s=1}^{S} b_{s} \prod_{k=1}^{M} J_{l_{k}}\left(\alpha a A_{k}\right)
$$

where $J_{l_{k}}$ is the Bessel function of order $l_{k}$. Note that the output amplitude of the desired carrier at frequency $\omega_{p}$ is given by $M\left(L_{p}\right)$ with $L_{p}=\{\ldots, 0, \underset{p: t h}{1}, 0, \ldots\}$.

\section{The Far Field Radiation Pattern}

Now consider the received power at the MS in direction $\theta$ from broadside of the array and on a distance $r$ from the BS. Assume an $N$ element, $\lambda / 2$-spaced linear array, where $\lambda$ is the wavelength of the carrier frequency in the center frequency channel. Furthermore we assume a simple fixed beamformer, which can be implemented as the fast Fourier transform (FFT), or if implemented in hardware, a Butler matrix. The phase of the beamformer "weight" applied to signal $k$ at antenna $n$ is denoted $\varphi_{k n}$. The far field radiated power in the direction $\theta$ of the $L_{p}$ :th signal component can then be calculated as

$$
\begin{aligned}
& P_{E I R P}\left(L_{p}\right)= \\
= & \left|M\left(L_{p}\right) \Omega(\theta) \sum_{n=1}^{N} e^{j \sum_{k=1}^{M}\left(l_{k} \varphi_{k n}-(n-1) \pi \sin (\theta)\right)}\right|^{2}
\end{aligned}
$$

where $\Omega(\theta)$ is the individual antenna element gain in direction $\theta$. Using the FFT or the Butler matrix, we have the property $\varphi_{k n}=(n-1) \Delta \varphi_{k}$ [5], where $\Delta \varphi_{k}$ is the phase gradient over the $N$ antennas in the array for signal $k$. The phase gradient $\Delta \varphi_{k}$ belongs to a set $\Omega_{S}$, of $N$ different phase gradients, unique for each beam the beamforming network can generate.

$$
\Omega_{S}=\left\{\frac{2 \pi p}{N}\right\}_{p=0}^{N-1}
$$

The finite set $\Omega_{S}$ is a closed group under multiplication and addition, following modulo- $N$ algebra. These properties implies that the main-lobe direction of the desired signals are also main-lobe directions for the IMD (if no $\mathrm{AM} / \mathrm{PM}$ amplifier conversion is assumed).

Using the FFT beamforming, (9) is simplified to

$$
P_{E I R P}\left(L_{p}\right)=\left|M\left(L_{p}\right) \Omega(\theta)\right|^{2} \frac{\sin ^{2}(N \xi(\theta))}{\sin ^{2}(\xi(\theta))}
$$

where

$$
\xi(\theta)=\frac{1}{2} \sum_{k=1}^{M}\left(l_{k} \Delta \varphi_{k}-\pi \sin (\theta)\right) .
$$

For example, the direction of the maximum radiated IMP for a $2 \omega_{j}-\omega_{k}$ product can be found as

$$
\theta_{m a x, I M P}=\arcsin \left(\frac{2 \Delta \varphi_{j}-\Delta \varphi_{k}}{\pi}\right) .
$$

It is easily verified that (13) maximizes (11) for the $2 \omega_{j}-$ $\omega_{k}$ product.

\section{SIMULATION SETUP}

The purpose of the simulation is to calculate the cumulative distribution function of the CIR at the MS in the fully loaded system. We simulate with a cluster size $K \in 1,4,7$ and a first tier of interfering BS. Each cell shape is assumed to be hexagonal and the MS are uniformly area distributed within a cell. We assume that a four element antenna array are employed in each sector $(N=4)$. The antenna elements have a $\cos ^{2}(\theta)$ radiation pattern with $\pm 90^{\circ}$ sensitivity (the front to back ratio is $\infty \mathrm{dB}$ ).

The power received at the MS from the BS transmitting at power $P_{E I R P}$ is, after averaging over the fast multipath fading,

$$
P_{r}=P_{E I R P}\left(\frac{1}{r}\right)^{\gamma} \Lambda
$$

where $r$ is the BS to MS distance, $\gamma$ is the path loss exponent (set to 4 ) and $\Lambda$ is a lognormal distributed random variable with mean $0 \mathrm{~dB}$ and standard deviation $4 \mathrm{~dB}$.

The MS are randomly placed in each cell and the MS used for the CIR estimation is picked from the center cell, to avoid edge effects. The beam with the highest received signal strength (RSSI) on the uplink for one particular user is chosen as the beam for the downlink transmission and the power is regulated according to (1). The users can be separated by using e.g. a training sequence method. 


\section{A. The Transmit MCPA}

The BS is assumed to be equipped with MCPA:s capable of amplifying a maximum of eight simultaneous carriers. The number of used frequency channels per cell is 12,21 and 24 in the $K=7,4,1$ cases respectively. As a measure of the linearity of the MCPA, the noise power ratio (NPR) is defined as the ratio between the linear output power density to the spectrum density within a pre-located notch[3]. When the number of co-amplified carriers is small (e.g. four), we use the intermodulation distortion ratio (IMR) as a linearity measure instead, due to the large errors invoked when removing one carrier in the NPR measurement. IMR is defined as the ratio between linear output power per tone to the highest intermodulation distortion power, assuming that all input carriers are of equal amplitude but with independent phases.

The AM/PM distortion is neglected in this study, it is often is small in a linearized amplifier, but the amplifier $\mathrm{AM} / \mathrm{AM}$ is modeled according to Cann's model [10]

$$
g(A)=\frac{\nu A}{\left(1+\left(\frac{\nu A}{K_{a}}\right)^{q}\right)^{\frac{1}{q}}}
$$

where $\nu$ is the small signal amplification, $K_{a}$ is the output saturation level as the input amplitude $|A| \rightarrow \infty$ and $q$ controls the knee sharpness, or the transition smoothness between the linear and nonlinear region. In the simulations, the values $q=10, K_{a}=1$ are used. The input backoff, defined as the average input power compared to the input saturation point, is adjusted to give the desired NPR.

\section{B. Performance Criteria}

To evaluate the performance of the switched beam antenna system, the area averaged probability of a received CIR at a MS exceeding a target value $\Gamma_{T}$. The outage probability as the probability that CIR is below a pre-defined power protection ratio. A power protection ratio of $15 \mathrm{~dB}$ were used in the simulations.

\section{SIMULATION RESULTS}

Fig. 2 and Fig. 3 shows the performance of the $K=7$ and $K=4$ system respectively for different NPR. Four carriers are co-amplified in each MCPA. The degradation due to the intermodulation distortion is similar in the $K=4$ cluster size, although we have a degradation in CIR compared to $K=7$ due to the reduced reuse distance and hence increased interference levels, as can be seen as a shift of the curves to the left in Fig. 3. The interference in these cases are linear co-channel interference transmitted by the BS in the first tier and intermodulation distortion from the BS in the same sector as the MS is connected to. We see that when NPR is decreased, the interference is dominated by intermodulation distortion.

When the reuse distance is reduced to $K=1$, see Fig. 4, the $\mathrm{NPR}=41 \mathrm{~dB}$ coincide with the ideal MCPA curve for low CIR. This implies that the linear interference from the $\mathrm{BS}$ in the first tier is the dominating interference. When NPR is decreased, the amplifier is working closer to the saturation point and the interference becomes more dominated by intermodulation distortion. Fig. 5 shows a

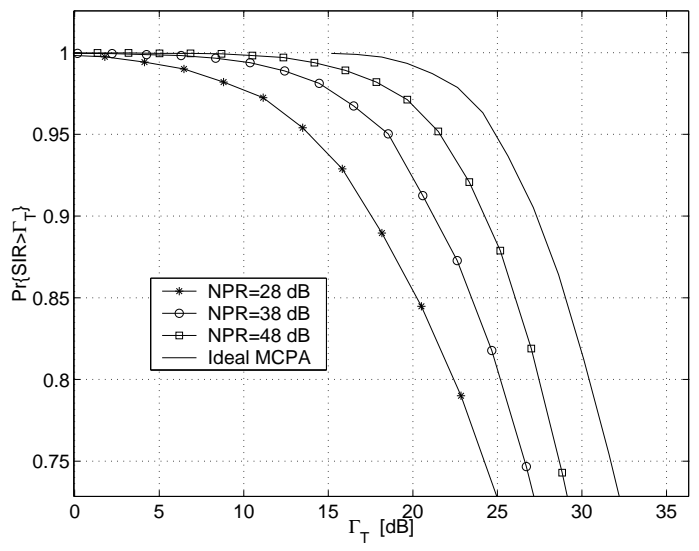

Figure 2: Area-averaged probability for different MCPA linearities measured as NPR. The curves shows the downlink with 4-element antenna arrays in each sector and cluster size $\mathrm{K}=7$.

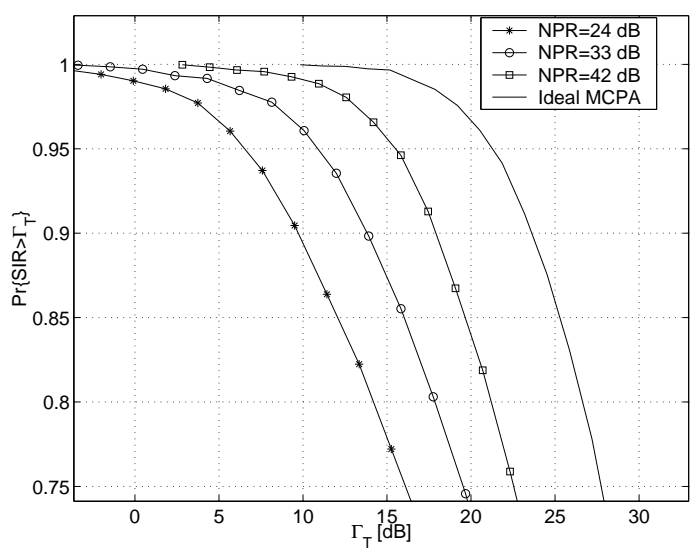

Figure 3: Area-averaged probability for different MCPA linearities measured as NPR. The curves shows the downlink with 4-element antenna arrays in each sector and cluster size $\mathrm{K}=4$.

comparison of the outage probabilities for the three cluster sizes considered. We see that the $K=4$ system is slightly more sensitive to intermodulation than the $K=7$ system, due to the reduced reuse distance, hence when NPR is decreased, a MS can be interfered by intermodulation from BS in the first tier. Note that the asymptotic value of the outage probability for the $K=1$ case is 0.79 when NPR goes to infinity, hence the system is interference limited due to the short reuse distance, even with ideal MCPA:s.

\section{CONCLUSION}

The performance of a switched multibeam antenna system using transmit MCPA:s in a cellular FDMA network has 


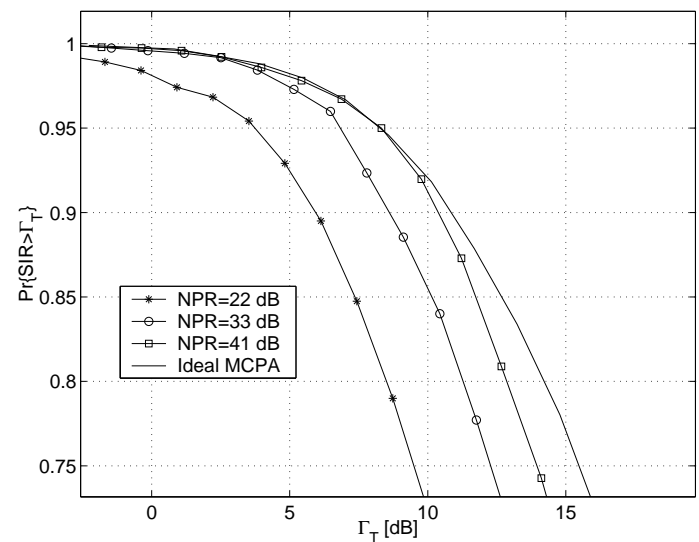

Figure 4: Area-averaged probability for different MCPA linearities measured as NPR. The curves shows the downlink with 4-element antenna arrays in each sector and cluster size $\mathrm{K}=1$.

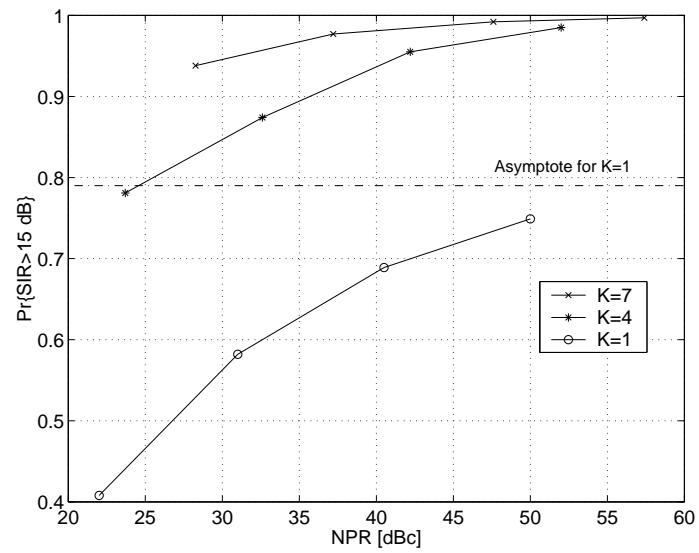

Figure 5: 1-Pr\{Outage $\}$ for different cluster sizes as a function of MCPA linearity.

been studied by simulations on the downlink channel. The IMP will be spatially filtered by the array radiation pattern and is often radiated in another direction than the direction where the two, or the three signals that generate the IMP are directed.

The multibeam antenna makes the system more robust to nonlinearities, for $K=7$, the CIR is severely reduced when the nonlinearity is present, by intermodulation distortion from the own BS. However, the multibeam antenna has increased the CIR to such an extent that the outage probability $\operatorname{Pr}\{C I R<15 d B\}$ is only slightly affected. For $K=1$ systems the linear interference situation is severe and every $\mathrm{dB}$ degradation in MS CIR is expensive, hence the outage probability will be largely affected when the NPR is decreased.

Hence, it was demonstrated that by using a multibeam antenna in $K=7$ frequency planned network, we can use the generated CIR overhead to use a less complex or less expensive linearisation method. To reduce the intermodulation distortion at the MS, it is possible allocate new mobiles to frequencies so that the generated intermodulation main beam points in directions where no mobile exists on that particular frequency channel.

\section{REFERENCES}

[1] M.D.Austin M.J.Ho, G.L.Stuber, "Performance of switched-beam smart antenna systems," IEEE Transactions on Vehicular Technology, vol. 47, pp. 10-19, 1998.

[2] J.McGeehan G.Tsoulos, M.Beach, "Space division multiple acess (SDMA) field trials. Part 2: Calibration and linearity issues," IEE Proc. - Radar, Sonar and Navigation, vol. 145, pp. 79-84, 1998.

[3] N.Borges de Carvalho J.Pedro, "On the use of multitone techniques for assessing RF components' intermodulation distortion," IEEE Transactions on $\mathrm{Mi}$ crowave Theory and Techniques, vol. 47, pp. 23932402, 1999.

[4] T.S.Rappaport P.Cardieri, "Combined effects of narrowbeam antennas and fractional loading factor in forward link cellular communication systems," in Proceedings of Vehicular Technology Conference, Piscataway, USA, 1999, pp. 1074-1078.

[5] M.Wennström, "Smart antenna implementation issues for wireless communications,", Technical report, Signals and Systems Group, Uppsala University, Sweden, 1999, Technical Licentiate Thesis, http://www.signal.uu.se/Publications/abstracts/1991.html.

[6] W.A. Sandrin, "Spatial distribution of intermodulation products in active phased array antennas," IEEE Transactions on Antennas and Propagation, vol. 22, pp. 864-868, 1973.

[7] M.Ljungberg C.Carneheim, S-O Jonsson, "FHGSM frequency hopping GSM," in Proceedings of Vehicular Technology Conference, Stockholm, Sweden, 1994, pp. 1155-1159.

[8] S.Sowelam K.J. Maalouf, R.C.Gaus, "Error rate estimation in a multi-channel active phased array," in IEEE International Conference on Communications, New York, USA, 1998, pp. 402-406.

[9] O.Shimbo, "Effects of intermodulation, AM-PM conversion, and additive noise in multicarrier TWT systems," Proceedings of the IEEE, vol. 59, no. 2, pp. 230-239, 1971.

[10] A.J.Cann, "Nonlinearity model with variable knee sharpness," IEEE Trans. on Aerospace and Electronic Systems, vol. 16, pp. 874-878, 1980. 